\title{
Lower bounds for testing complete positivity and quantum separability
}

\author{
Costin Bădescu* ～Ryan O’Donnell*
}

September 11, 2019

\begin{abstract}
In this work we are interested the problem of testing quantum entanglement. More specifically, we study the separability problem in quantum property testing, where one is given $n$ copies of an unknown mixed quantum state $\varrho$ on $\mathbb{C}^{d} \otimes \mathbb{C}^{d}$, and one wants to test whether $\varrho$ is separable or $\epsilon$-far from all separable states in trace distance. We prove that $n=\Omega\left(d^{2} / \epsilon^{2}\right)$ copies are necessary to test separability, assuming $\epsilon$ is not too small, viz. $\epsilon=\Omega(1 / \sqrt{d})$.

We also study completely positive distributions on the grid $[d] \times[d]$, as a classical analogue of separable states. We analogously prove that $\Omega\left(d / \epsilon^{2}\right)$ samples from an unknown distribution $p$ are necessary to decide whether $p$ is completely positive or $\epsilon$-far from all completely positive distributions in total variation distance. Towards showing that the true complexity may in fact be higher, we also show that learning an unknown completely positive distribution on $[d] \times[d]$ requires $\Omega\left(d^{2} / \epsilon^{2}\right)$ samples.
\end{abstract}

\section{Introduction}

A bipartite quantum state $\varrho$ on $\mathbb{C}^{d} \otimes \mathbb{C}^{d}$ is said to be separable if it can be written as a convex combination of product states, meaning states of the form $\rho_{1} \otimes \rho_{2}$ where $\rho_{1}$ and $\rho_{2}$ are quantum states on $\mathbb{C}^{d}$. Separable quantum states are precisely those states which do not exhibit any form of quantum entanglement. These are the only states that can be prepared

${ }^{*}$ Computer Science Department, Carnegie Mellon University. Supported by NSF grant FET-1909310. This material is based upon work supported by the National Science Foundation under grant numbers listed above. Any opinions, findings and conclusions or recommendations expressed in this material are those of the author and do not necessarily reflect the views of the National Science Foundation (NSF). $\{$ cbadescu, odonnell\}@ecs. cmu. edu 
by separated parties who can only share classical information. Understanding the general structure and properties of the set of separable states in higher dimensions is a difficult problem and is the subject of much ongoing research. For instance, deciding whether a given $d^{2} \times d^{2}$ matrix represents a separable state on $\mathbb{C}^{d} \otimes \mathbb{C}^{d}-$ also known as the separability problem in the quantum literature - is NP-hard [Gur04]. In this work, we study the following property testing version of the separability problem:

Provided unrestricted measurement access to $n$ copies of an unknown quantum state $\varrho$ on $\mathbb{C}^{d} \otimes \mathbb{C}^{d}$, decide with high probability if $\varrho$ is separable or $\epsilon$-far from all separable states in trace distance.

The ultimate goal is to determine the number of copies of $\varrho$ that is necessary and sufficient to solve the problem, up to constant factors, as a function of $d$ and $\epsilon$.

By estimating (i.e., fully learning) @ using recent algorithms for quantum state tomography $\left[\mathrm{HHJ}^{+} 16, \mathrm{OW} 16\right]$ and checking if the estimate is sufficiently close to a separable state, this problem can be solved using $O\left(d^{4} / \epsilon^{2}\right)$ copies of $\varrho$. In this paper, we prove a lower bound, showing that $\Omega\left(d^{2} / \epsilon^{2}\right)$ copies of $\varrho$ are necessary when $\epsilon=\Omega(1 / \sqrt{d})$; this reaches a lower bound of $\Omega\left(d^{3}\right)$ for $\epsilon=\Theta(1 / \sqrt{d})$. Closing the gap between the known bounds seems like a difficult problem, and we have no particularly strong feeling about whether the tight bound is the upper bound, the lower bound, or something in between. (Indeed, at least one paper [AS17a] contains some evidence that $\widetilde{\Theta}\left(d^{3}\right)$ might be the true complexity for constant $\epsilon$.)

Given the difficulty of closing the gap, we have sought a classical analogue of the separability testing problem to try as a first step. Analogies between quantum states and classical probability distributions have proven to be a helpful source of inspiration throughout quantum theory. Unfortunately, entanglement is understood to be a purely quantum phenomenon; every finitely-supported discrete distribution can be expressed as a convex combination of product point distributions, so there are no "entangled" distributions. But motivated by the characterization of separable quantum states using symmetric extensions and the quantum de Finetti theorem [DPS04], we propose as a kind of analogue the study of mixtures of i.i.d. bivariate distributions, which arise in the classical de Finetti theorem. Doherty et al. [DPS04] used the quantum de Finetti theorem to show that a quantum state $\varrho$ on $\mathbb{C}^{d} \otimes \mathbb{C}^{d}$ is separable (i.e. a mixture of product states) if and only if $\varrho$ has a symmetric extension to $\mathbb{C}^{d} \otimes\left(\mathbb{C}^{d}\right)^{\otimes k}$ for any positive integer $k$. Somewhat analogously, the classical de Finetti theorem states that a sequence of real random variables is a mixture of i.i.d. sequences of random variables if and only if it is exchangeable [Dia77].

We call distributions which are mixtures of i.i.d. bivariate distributions completely positive, due to their connection with completely positive matrices. We show that, given sample access to an unknown distribution $p$ over $[d] \times[d]$, at least $\Omega\left(d / \epsilon^{2}\right)$ samples are necessary to 
decide with high probability if $p$ is completely positive or $\epsilon$-far from all completely positive distributions in total variation distance. Our proof is a generalization of Paninski's lower bound for testing if a distribution is uniform [Pan08].

Regarding upper bounds, one can again get a trivial upper bound of $O\left(d^{2} / \epsilon^{2}\right)$ samples for testing complete positivity, simply by fully estimating $p$ to $\epsilon$-accuracy in total variation distance. We again do not know how to close the gap between $\Omega\left(d / \epsilon^{2}\right)$ and $O\left(d^{2} / \epsilon^{2}\right)$, but we present evidence that the upper bound may be the true complexity. Specifically, a common strategy for trying to test a family $D$ of distributions is to solve the problem of learning an unknown distribution promised to be in $D$. We show that learning a completely positive distribution on $[d] \times[d]$ to accuracy $\epsilon$ requires $\Omega\left(d^{2} / \epsilon^{2}\right)$ samples. On the other hand, we are not able to show an analogous improved lower bound for learning separable quantum states.

\subsection{Previous work}

The property testing version of the separability problem, as defined above, appears in [MdW16], where a lower bound of $\Omega\left(d^{2}\right)$ is proven for constant $\epsilon$. As in [MdW16], our proof also reduces the problem of testing if a state is separable to the problem of testing if a state is the maximally mixed state. However, we do not pass through the notion of entanglement of formation, as [MdW16] does, and instead rely on results about the convex structure of the set of separable states. This approach yields a more direct proof that certain random states are w.h.p. far from separable, which allows us to take advantage of a lower bound from [OW15] (see Theorem 4.1).

We believe that the separability testing problem has seen further study, but that there has been a lack of results due to its difficulty. There is a very extensive literature on the subject of entanglement detection (see e.g. [GT09, HHHH09]), which is concerned with establishing different criteria for detecting or verifying entanglement. However, it is not obvious how these results can be applied in the property testing setting. In particular, few of these criteria are specifically concerned with states that are far from separable in trace distance and many only apply to certain restricted classes of quantum states.

As regards our classical analogue - testing if a bipartite distribution is completely positive (mixture of i.i.d.) - we are not aware of previous work in the literature. The proof of our $\Omega\left(d / \epsilon^{2}\right)$ lower bound is inspired by, and generalizes, Paninski's lower bound for testing if a distribution is uniform [Pan08]. The proof of our tight $\Omega\left(d^{2} / \epsilon^{2}\right)$ lower bound for learning completely positive distributions uses the Fano inequality method. 


\subsection{Outline}

In Section 2 we cover background material on completely positive distributions, quantum states and separability, and the property testing framework that our results are concerned with. In Section 3, we prove that testing if a distribution $p$ on $[d] \times[d]$ is completely positive or $\epsilon$-far from all completely positive distributions in total variation distance requires $\Omega\left(d / \epsilon^{2}\right)$ samples from $p$; we also prove that learning completely positive distributions requires $\Omega\left(d^{2} / \epsilon^{2}\right)$ samples. Finally, in Section 4 , we show that testing if a quantum state $\varrho$ on $\mathbb{C}^{d} \otimes \mathbb{C}^{d}$ is separable or $\epsilon$-far from all separable states in trace distance requires $\Omega\left(d^{2} / \epsilon^{2}\right)$ copies of $\varrho$ when $\epsilon=\Omega(1 / \sqrt{d})$.

\section{Preliminaries}

This section covers the mathematical background and notation used in the rest of the paper.

\subsection{Completely positive distributions}

There is a well-developed theory of completely positive and copositive matrices (see e.g. [GM12, Chapter 7]). In this section, we review some known material.

Let $d$ be a positive integer. We consider distributions over the grid $[d]^{2}=[d] \times[d]=$ $\{(1,1),(1,2), \ldots,(d, d)\}$ which we represent as matrices $A \in \mathbb{R}^{d \times d}$ with $A_{i j}$ being the probability of sampling $(i, j)$.

Example 2.1. If $p \in \mathbb{R}^{d}$ is a distribution on $[d]=\{1, \ldots, d\}$ represented as a column vector, then $p p^{\top}$ is the natural i.i.d. product probability distribution on $[d] \times[d]$ derived from $p$, with $p_{i} p_{j}$ being the probability of sampling $(i, j)$.

Definition 2.2. A matrix $A \in \mathbb{R}^{d \times d}$ is completely positive (CP) if there exist vectors $v_{1}, \ldots, v_{k} \in \mathbb{R}_{\geq 0}^{d}$ with nonnegative entries such that $A$ can be expressed as a convex combination of their projections $v_{1} v_{1}^{\top}, \ldots, v_{k} v_{k}^{\top}$, viz.

$$
A=\sum_{i=1}^{k} c_{i} v_{i} v_{i}^{\top}
$$

for some nonnegative real numbers $c_{1}, \ldots, c_{k} \in \mathbb{R}$ with $c_{1}+\cdots+c_{k}=1$.

A distribution on $[d]^{2}$ represented as a matrix $A$ is completely positive if $A$ is a CP matrix.

Remark 2.3. For a CP distribution $A$, the vectors $v_{i}$ in Equation (1) may be taken to be probability distributions, since one can replace $v_{i}$ by $v_{i} /\left\|v_{i}\right\|_{1}$ and $c_{i}$ by $c_{i}\left\|v_{i}\right\|_{1}^{2}$. Thus, CP distributions are precisely the mixtures of i.i.d. distributions. 
It follows immediately from Definition 2.2 that a $\mathrm{CP}$ matrix $A$ satisfies three basic properties:

(i) $A$ is symmetric $\left(A^{\top}=A\right)$,

(ii) $A_{i j} \geq 0$ for all $i, j \in[d]$, and

(iii) $A$ is positive semidefinite (PSD), denoted $A \geq 0$.

A matrix satisfying these three properties is called doubly nonnegative. However, if $d \geq 5$, then there exist doubly nonnegative matrices which are not completely positive [MM62].

Example 2.4. Let $J$ denote the $d \times d$ matrix with $J_{i j}=1$ for all $i, j \in[d]$ and let Unif $d^{2}=$ $J / d^{2}$ denote the uniform distribution on $[d]^{2}$. Since Unif $d^{2}=\left(\frac{1}{d}, \ldots, \frac{1}{d}\right)\left(\frac{1}{d}, \ldots, \frac{1}{d}\right)^{\top}$, the uniform distribution on $[d]^{2}$ is completely positive.

Let $\mathrm{CP}_{d}$ denote the set of completely positive $d \times d$ matrices and let $\mathrm{CPD}_{d}$ denote its subset of completely positive distributions on $[d]^{2}$. It is well known that $\mathrm{CP}_{d}$ is a cone and that its dual cone consists of copositive matrices, i.e. matrices $M$ such that $x^{\top} M x \geq 0$ for all nonnegative vectors $x \in \mathbb{R}_{>0}^{d}$. Thus, by cone duality, if $B \notin \mathrm{CP}_{d}$ is a non-CP matrix, then there exists a copositive matrix $W$ such that $\operatorname{tr}(A W) \geq 0$ for all $A \in \mathrm{CP}_{d}$ and $\operatorname{tr}(B W)<0$. This result yields witnesses certifying nonmembership in $\mathrm{CPD}_{d}$. However, its usefulness is limited by the fact that it provides no quantitative information about how far a nonmember $A$ is from the set $\mathrm{CPD}_{d}$.

In what follows, we interpret distributions on $[d]^{2}$ as weighted directed graphs with selfloops and obtain a sufficient condition for a distribution to be $\epsilon$-far in total variation distance from $\mathrm{CPD}_{d}$ in terms of the maximum value of a cut in the corresponding graph.

We interpret a distribution $A$ on $[d]^{2}$ as a weighted directed graph $G$ with vertices $V(G)=$ $[d]$ and edges

$$
E(G)=\left\{(i, j) \in[d]^{2} \mid A_{i j}>0\right\} .
$$

A cut $x \in\{ \pm 1\}^{d}$ in $G$ is a bipartition of the vertices $V(G)=E_{1} \cup E_{2}$ with $E_{1}=\{i \in[d] \mid$ $\left.x_{i}<0\right\}$ and $E_{2}=\left\{i \in[d] \mid x_{i}>0\right\}$. The total weight of edges cut by this bipartition is

$$
\sum_{(i, j) \in[d]^{2}} \frac{1-x_{i} x_{j}}{2} A_{i j}=\underset{(\boldsymbol{i}, \boldsymbol{j}) \sim A}{\mathbf{E}} \frac{1-x_{\boldsymbol{i}} x_{\boldsymbol{j}}}{2}=\frac{1}{2}-\frac{1}{2} \underset{(\boldsymbol{i}, \boldsymbol{j}) \sim A}{\mathbf{E}} x_{\boldsymbol{i}} x_{\boldsymbol{j}}=\frac{1}{2}-\frac{1}{2} x^{\top} A x .
$$

In particular, if $A=p p^{\top}$ with $p \in \mathbb{R}^{d}$, then

$$
x^{\top} A x=x^{\top} p p^{\top} x=\left(x^{\top} p\right)^{2} \geq 0 .
$$

By Remark 2.3, a CP distribution is a convex combination of matrices of the form $p p^{\top}$. Thus, the following holds: 
Proposition 2.5. If $A$ is a CP distribution, then the total weight of a cut in the graph represented by $A$ is at most $\frac{1}{2}$.

This fact allows us to prove the following result which gives a sufficient condition for a distribution to be $\epsilon$-far from all $\mathrm{CP}$ distributions in $\ell^{1}$ distance. (The matrix norms in the following are entrywise.)

Proposition 2.6. Let $A$ be a distribution on $[d]^{2}$. If there exists a cut $x \in\{ \pm 1\}^{d}$ with $x^{T} A x \leq-\epsilon$, then $\|B-A\|_{1} \geq \epsilon$ for all $B \in \mathrm{CPD}_{d}$.

Proof. Let $B \in \mathrm{CPD}_{d}$ be arbitrary. By Hölder's inequality, for all $U \in \mathbb{R}^{d \times d}$ with $\|U\|_{\infty}=1$,

$$
\|B-A\|_{1} \geq \operatorname{tr}\left(U^{\top}(B-A)\right)=\operatorname{tr}\left(U^{\top} B\right)-\operatorname{tr}\left(U^{\top} A\right)
$$

Let $U=x x^{\top}$. Since $x^{\top} B x \geq 0$ and $\operatorname{tr}\left(U^{\top} A\right)=x^{\top} A x \leq-\epsilon$,

$$
\|B-A\|_{1} \geq x^{\top} B x-x^{\top} A x \geq \epsilon .
$$

\subsection{Quantum states and separability}

This section serves as a brief introduction to quantum states and separability. For a more comprehensive introduction, see e.g. [Wat18].

We work over $\mathbb{C}$ and use bra-ket notation to denote vectors in $\mathbb{C}^{d}$, viz. for all vectors $x, y \in \mathbb{C}^{d}$ and matrices $A \in \mathbb{C}^{d \times d},|x\rangle=x,\langle x|=x^{\dagger}=\bar{x}^{\top},\langle x \otimes y|=\langle x|\otimes\langle y||, x \otimes y\rangle=| x\rangle \otimes| y\rangle$, $\langle x \mid y\rangle=x^{\dagger} y,|x\rangle\langle y|=x y^{\dagger}$, and $\langle x|A| y\rangle=x^{\dagger} A y$.

Definition 2.7. A quantum state $\rho$ on $\mathbb{C}^{d}$ is a positive semidefinite matrix $\rho \in \mathbb{C}^{d \times d}$ with $\operatorname{tr}(\rho)=1$. A measurement is a set $\left\{E_{1}, \ldots, E_{k}\right\}$ of positive semidefinite matrices on $\mathbb{C}^{d}$ with $E_{1}+\cdots+E_{k}=\mathbb{1}$, where $\mathbb{1}$ denotes the identity matrix.

Let $\rho$ and $\left\{E_{1}, \ldots, E_{k}\right\}$ be as in the definition above and let $p_{i}=\operatorname{tr}\left(\rho E_{i}\right)$ for $i=1, \ldots, k$. Since $\rho$ and the $E_{i}$ are PSD, $p_{i} \geq 0$ for all $i=1, \ldots, k$, and

$$
p_{1}+\cdots+p_{k}=\operatorname{tr}\left(\rho E_{1}\right)+\cdots+\operatorname{tr}\left(\rho E_{k}\right)=\operatorname{tr}\left(\rho\left(E_{1}+\cdots+E_{k}\right)\right)=\operatorname{tr}(\rho)=1 .
$$

Hence, $\left(p_{1}, \ldots, p_{k}\right)$ is a distribution on $[k]$. Applying the measurement $\left\{E_{1}, \ldots, E_{k}\right\}$ to the quantum state $\rho$ yields outcome $i \in[k]$ with probability $p_{i}=\operatorname{tr}\left(\rho E_{i}\right)$.

Example 2.8. $\frac{\mathbb{1}}{d}$ is a quantum state on $\mathbb{C}^{d}$ called the maximally mixed state; it is analogous to the uniform distribution on $[d]$.

Definition 2.9. A state of the form $\rho=|x\rangle\langle x|$ for some $x \in \mathbb{C}^{d}$ is called a pure state. 
Given quantum states $\rho$ and $\sigma$ on $\mathbb{C}^{d}$, the tensor product $\rho \otimes \sigma$ is a quantum state on $\mathbb{C}^{d} \otimes \mathbb{C}^{d}$. If $\rho$ and $\sigma$ represent the individual states of two isolated particles, then $\rho \otimes \sigma$ is the state of the physical system comprising both particles. Thus, the system composed of $n$ identical copies of the state $\rho$ is represented as the state $\rho^{\otimes n}$ on $\left(\mathbb{C}^{d}\right)^{\otimes n}$.

Definition 2.10. A quantum state $\varrho$ on $\mathbb{C}^{d} \otimes \mathbb{C}^{d}$ is separable if $\varrho$ can be expressed as a convex combination of product states, viz.

$$
\varrho=\sum_{i=1}^{k} c_{i} \rho_{i} \otimes \sigma_{i},
$$

where $\rho_{i}$ and $\sigma_{i}$ are states on $\mathbb{C}^{d}$ for $i=1, \ldots, k$ and $c_{1}, \ldots, c_{k} \in \mathbb{R}_{\geq 0}$ satisfy $c_{1}+\ldots+c_{k}=1$. Thus, the physical system represented by $\varrho$ may be regarded as being in the state $\rho_{i} \otimes \sigma_{i}$ with probability $c_{i}$.

A state that is not separable is called entangled.

Example 2.11. Since $\frac{\mathbb{1}}{d^{2}}=\frac{\mathbb{1}}{d} \otimes \frac{\mathbb{1}}{d}$, the maximally mixed state is separable.

Definition 2.12. Let Sep denote the set of separable states on $\mathbb{C}^{d} \otimes \mathbb{C}^{d}$ and let Sep $\mathrm{p}_{ \pm}$denote its cylindrical symmetrization (cf. [AS17b, p. 81]), viz. $\operatorname{Sep}_{ \pm}=\operatorname{conv}(\operatorname{Sep} \cup(-\operatorname{Sep}))$, where $\operatorname{conv}(E)$ denotes the convex hull of the set $E$.

Similar to the duality between completely positive and copositive matrices, the set Sep generates a cone of separable operators whose dual is the cone of block-positive operators (see e.g. $[\mathrm{AS} 17 \mathrm{~b}]$ ). A block-positive operator acts as an entanglement witness certifying that a given quantum state is not separable. Thus, Proposition 4.5 in Section 4 is comparable to Proposition 2.6 in that it describes witnesses certifying that a quantum state is not just entangled but actually $\epsilon$-far from all separable states in trace distance.

\subsection{The property testing framework}

In the property testing model, we have a set $\mathcal{O}$ of objects and also a distance function dist : $\mathcal{O} \times \mathcal{O} \rightarrow \mathbb{R}$. A property $\mathcal{P}$ is a subset of $\mathcal{O}$ and the distance between an object $x \in \mathcal{O}$ and the property $\mathcal{P}$ is defined by $\operatorname{dist}(x, \mathcal{P})=\inf _{y \in \mathcal{P}} \operatorname{dist}(x, y)$. An algorithm $\mathcal{T}$ is said to test $\mathcal{P}$ if, given some type of access to $x \in \mathcal{O}$ (e.g. independent samples or identical copies), $\mathcal{T}$ accepts $x$ w.h.p. when $x \in \mathcal{P}$ and $\mathcal{T}$ rejects $x$ w.h.p. when $\operatorname{dist}(x, \mathcal{P}) \geq \epsilon$.

In Section 3, $\mathcal{O}$ is the set of distributions on $[d] \times[d]$, dist is the total variation distance, and $\mathcal{P}=\mathrm{CPD}_{d} \subseteq \mathcal{O}$ is the set of $\mathrm{CP}$ distributions. Given samples $\boldsymbol{x}_{1}, \ldots, \boldsymbol{x}_{n}$ from a distribution $p$ on $[d]^{2}$, a testing algorithm $\mathcal{T}$ for $\mathrm{CPD}_{d}$ satisfies

$$
p \in \mathrm{CPD}_{d} \Longrightarrow \mathbf{P}\left[\mathcal{T}\left(\boldsymbol{x}_{1}, \ldots, \boldsymbol{x}_{n}\right) \text { accepts }\right] \geq \frac{2}{3},
$$




$$
p \epsilon \text {-far from } \mathrm{CPD}_{d} \Longrightarrow \mathbf{P}\left[\mathcal{T}\left(\boldsymbol{x}_{1}, \ldots, \boldsymbol{x}_{n}\right) \text { accepts }\right] \leq \frac{1}{3}
$$

In Section $4, \mathcal{O}$ is the set of quantum states on $\mathbb{C}^{d} \otimes \mathbb{C}^{d}$, $\operatorname{dist}(\varrho, \sigma)=\frac{1}{2}\|\varrho-\sigma\|_{1}$ is the trace distance between quantum states, and $\mathcal{P}=$ Sep is the set of separable states on $\mathbb{C}^{d} \otimes \mathbb{C}^{d}$. Given measurement access to $n$ copies $\varrho^{\otimes n}$ of a state $\varrho \in \mathbb{C}^{d} \otimes \mathbb{C}^{d}$, a testing algorithm for Sep is a two-outcome measurement $\left\{E_{0}, E_{1}\right\}$ on $\left(\mathbb{C}^{d}\right)^{\otimes n}$ satisfying:

$$
\begin{array}{r}
\left.\varrho \in \operatorname{Sep} \Longrightarrow \operatorname{tr}\left(E_{1} \varrho^{\otimes n}\right)=1\right] \geq \frac{2}{3}, \\
\left.\varrho \in \text {-far from Sep } \Longrightarrow \operatorname{tr}\left(E_{1} \varrho^{\otimes n}\right)=1\right] \leq \frac{1}{3} .
\end{array}
$$

\section{Testing complete positivity}

Let $d$ be a positive integer. If $d$ is odd, we can reduce to the case of $d-1$ by using distributions that don't involve outcome $d \in[d]$, and the asymptotics of $\Omega\left(d / \epsilon^{2}\right)$ remain unchanged. Hence we may assume, without loss of generality, that $d$ is even.

We begin by defining a family of distributions on $[d]^{2}$ which are $\epsilon$-far from $\mathrm{CPD}_{d}$. Let $S \subseteq[d]$ be a subset of size $|S|=\frac{d}{2}$. Thus, $\left|S^{\mathrm{c}}\right|=\frac{d}{2}$ and

$$
\left|S \times S^{\mathrm{c}} \cup S^{\mathrm{c}} \times S\right|=\left|S \times S^{\mathrm{c}}\right|+\left|S^{\mathrm{c}} \times S\right|=\frac{d^{2}}{2} .
$$

Let $\phi_{S}:[d]^{2} \rightarrow \mathbb{R}$ be the function defined by

$$
\phi_{S}(x)= \begin{cases}1+\epsilon, & x \in S \times S^{\mathrm{c}} \cup S^{\mathrm{c}} \times S \\ 1-\epsilon, & \text { otherwise. }\end{cases}
$$

Hence,

$$
\underset{x \in[d]^{2}}{\operatorname{avg}} \phi_{S}(x)=\frac{1}{d^{2}}\left(\frac{d^{2}}{2}(1+\epsilon)+\frac{d^{2}}{2}(1-\epsilon)\right)=1 .
$$

So we may think of $\phi_{S}$ as a density function with respect to the uniform distribution on $[d]^{2}$.

Let $x \in\{ \pm 1\}^{d}$ be defined as follows: for all $i \in[d]$, if $i \in S$, then $x_{i}=1$, otherwise $x_{i}=-1$. Let $A^{S}$ be the matrix defined by $A_{i j}^{S}=\phi_{S}((i, j)) / d^{2}$. Thus, $A^{S}$ is a symmetric distribution on $[d]^{2}$ and $x$ is a cut. The total weight of this cut is

$$
\frac{d^{2}}{2} \cdot \frac{1+\epsilon}{d^{2}}=\frac{1}{2}+\frac{\epsilon}{2} .
$$


Therefore, for every subset $S \subseteq[d]$, the distribution $A^{S}$ is not completely positive. Moreover, $x^{\top} A^{S} x=-\epsilon$, so, by Proposition 2.6,

$$
\left\|A^{S}-B\right\|_{1} \geq \epsilon
$$

for every $\mathrm{CP}$ distribution $B$ (where the matrix norm is entry-wise). In other words, for every subset $S \subseteq[d]$ with $|S|=\frac{d}{2}, A^{S}$ is a distribution on $[d]^{2}$ which is $\epsilon$-far in $\ell^{1}$ distance from every CP distribution on $[d]^{2}$.

Fix $\Omega=[d]^{2}$ and let $\phi: \Omega^{n} \rightarrow \mathbb{R}$ denote the function defined by

$$
\phi(x)=\operatorname{avg}_{\substack{S \subseteq[d] \\|S|=d / 2}} \phi_{S}\left(x_{1}\right) \cdots \phi_{S}\left(x_{n}\right) .
$$

Let $\mathcal{D}_{n}$ denote the distribution on $\Omega^{n}$ defined by the density $\phi$ and let $d_{\chi^{2}}\left({ }_{-},,_{-}\right)$denote the $\chi^{2}$-distance between probability distributions, i.e. for distributions $\mathcal{P}$ and $\mathcal{Q}$ on $\Omega$,

$$
d_{\chi^{2}}(\mathcal{P}, \mathcal{Q})=\underset{\boldsymbol{x} \sim \mathcal{Q}}{\mathbf{E}}\left[\left(\frac{\mathcal{P}(\boldsymbol{x})}{\mathcal{Q}(\boldsymbol{x})}-1\right)^{2}\right] .
$$

The following proposition will be shown to imply our lower bound:

Proposition 3.1. If $d_{\chi^{2}}\left(\mathcal{D}_{n}\right.$, Unif $\left._{d^{2}}^{\otimes n}\right) \geq \frac{1}{3}$, then $n=\Omega\left(d / \epsilon^{2}\right)$.

Proof. Let $\mathcal{H}$ denote the uniform distribution over subsets $S \subseteq[d]$ with $|S|=d / 2$. Thus,

$$
\begin{aligned}
d_{\chi^{2}}\left(\mathcal{D}_{n}, \text { Unif }_{d^{2}}^{\otimes n}\right) & =\left(\sum_{x \in \Omega^{n}} \frac{\mathcal{D}_{n}(x)^{2}}{\operatorname{Unif}_{d^{2}}^{\otimes n}(x)}\right)-1 \\
& =\left(\sum_{x \in \Omega^{n}} \frac{\phi(x)^{2}}{d^{2 n}}\right)-1 \\
& =\underset{\boldsymbol{x} \sim \mathrm{Unif}_{d^{2}}^{\otimes n}}{\mathbf{E}} \phi(\boldsymbol{x})^{2}-1 \\
& =\underset{\boldsymbol{x} \sim \mathrm{Unif}_{d^{2}}^{\otimes n}}{\mathbf{E}}\left[\left(\underset{\boldsymbol{S} \sim \mathcal{H}}{\mathbf{E}} \phi_{\boldsymbol{S}}\left(\boldsymbol{x}_{1}\right) \cdots \phi_{\boldsymbol{S}}\left(\boldsymbol{x}_{n}\right)\right)^{2}\right]-1 \\
& =\underset{\boldsymbol{x} \sim \mathrm{Unif}_{d^{2}}^{\otimes n}}{\mathbf{E}}\left[\underset{\boldsymbol{S}, \boldsymbol{S}^{\prime} \sim \mathcal{H}}{\mathbf{E}} \phi_{\boldsymbol{S}}\left(\boldsymbol{x}_{1}\right) \cdots \phi_{\boldsymbol{S}}\left(\boldsymbol{x}_{n}\right) \phi_{\boldsymbol{S}^{\prime}}\left(\boldsymbol{x}_{1}\right) \cdots \phi_{\boldsymbol{S}^{\prime}}\left(\boldsymbol{x}_{n}\right)\right]-1 \\
& =\underset{\boldsymbol{S}, \boldsymbol{S}^{\prime} \sim \mathcal{H}}{\mathbf{E} \sim \mathrm{Unif}_{d^{2}}^{\otimes n}} \phi_{\boldsymbol{S}}\left(\boldsymbol{x}_{1}\right) \cdots \phi_{\boldsymbol{S}}\left(\boldsymbol{x}_{n}\right) \phi_{\boldsymbol{S}^{\prime}}\left(\boldsymbol{x}_{1}\right) \cdots \phi_{\boldsymbol{S}^{\prime}}\left(\boldsymbol{x}_{n}\right)-1
\end{aligned}
$$




$$
=\underset{\boldsymbol{S}, \boldsymbol{S}^{\prime} \sim \mathcal{H}}{\mathbf{E}}\left[\left(\underset{\boldsymbol{x} \sim \mathrm{Unif}_{d^{2}}}{\mathbf{E}} \phi_{\boldsymbol{S}}(\boldsymbol{x}) \phi_{\boldsymbol{S}^{\prime}}(\boldsymbol{x})\right)^{n}\right]-1 .
$$

For a subset $E \subseteq[d]$, let $\chi_{E}$ be the \pm 1 -valued indicator function defined by $\chi_{E}(x)=1$ if $x \in E$ and $\chi_{E}(x)=-1$ otherwise. Note that $\phi_{E}(x)=1-\chi_{E}\left(x_{1}\right) \chi_{E}\left(x_{2}\right) \epsilon$ for all $x \in \Omega$. Hence,

$$
\phi_{\boldsymbol{S}}(\boldsymbol{x}) \phi_{\boldsymbol{S}^{\prime}}(\boldsymbol{x})=1-\left(\chi_{\boldsymbol{S}}\left(\boldsymbol{x}_{1}\right) \chi_{\boldsymbol{S}}\left(\boldsymbol{x}_{2}\right)+\chi_{\boldsymbol{S}^{\prime}}\left(\boldsymbol{x}_{1}\right) \chi_{\boldsymbol{S}^{\prime}}\left(\boldsymbol{x}_{2}\right)\right) \epsilon+\chi_{\boldsymbol{S}}\left(\boldsymbol{x}_{1}\right) \chi_{\boldsymbol{S}}\left(\boldsymbol{x}_{2}\right) \chi_{\boldsymbol{S}^{\prime}}\left(\boldsymbol{x}_{1}\right) \chi_{\boldsymbol{S}^{\prime}}\left(\boldsymbol{x}_{2}\right) \epsilon^{2} .
$$

For a fixed outcome of $\boldsymbol{S}$ and $\boldsymbol{x}$ uniformly random, $\chi_{\boldsymbol{S}}\left(\boldsymbol{x}_{1}\right)$ and $\chi_{\boldsymbol{S}}\left(\boldsymbol{x}_{2}\right)$ are independent uniform \pm 1 -valued bits. So, in expectation, the terms involving just $\epsilon$ in the expression above drop out. Moreover, $\chi_{\boldsymbol{S}}\left(\boldsymbol{x}_{1}\right) \chi_{\boldsymbol{S}^{\prime}}\left(\boldsymbol{x}_{1}\right)$ and $\chi_{\boldsymbol{S}}\left(\boldsymbol{x}_{2}\right) \chi_{\boldsymbol{S}^{\prime}}\left(\boldsymbol{x}_{2}\right)$ are independent. Hence,

$$
\underset{\boldsymbol{x} \sim \mathrm{Unif}_{d^{2}}}{\mathbf{E}} \phi_{\boldsymbol{S}}(\boldsymbol{x}) \phi_{\boldsymbol{S}^{\prime}}(\boldsymbol{x})=1-\epsilon^{2} \cdot\left(\underset{\boldsymbol{x} \sim \mathrm{Unif}_{d^{2}}}{\mathbf{E}} \chi_{\boldsymbol{S}}\left(\boldsymbol{x}_{1}\right) \chi_{\boldsymbol{S}^{\prime}}\left(\boldsymbol{x}_{1}\right)\right)^{2}
$$

Let $\boldsymbol{r}=\left|\boldsymbol{S} \cap \boldsymbol{S}^{\prime}\right|$, where $\boldsymbol{S}, \boldsymbol{S}^{\prime} \sim \mathcal{H}$, and let $\boldsymbol{\delta}$ denote the mean of $\chi_{\boldsymbol{S}}\left(\boldsymbol{x}_{1}\right) \chi_{\boldsymbol{S}^{\prime}}\left(\boldsymbol{x}_{1}\right)$ appearing above. It is easy to check that $\boldsymbol{\delta}=4 \boldsymbol{r} / d-1$. Thus,

$$
\begin{aligned}
d_{\chi^{2}}\left(\mathcal{D}_{n}, \text { Unif }_{d^{2}}^{\otimes n}\right) & \leq \underset{\boldsymbol{S}, \boldsymbol{S}^{\prime} \sim \mathcal{H}}{\mathbf{E}}\left[\left(1+\epsilon^{2} \boldsymbol{\delta}^{2}\right)^{n}\right]-1 \\
& \leq \underset{\boldsymbol{S}, \boldsymbol{S}^{\prime} \sim \mathcal{H}}{\mathbf{E}}\left[\exp \left(\epsilon^{2} \boldsymbol{\delta}^{2}\right)^{n}\right]-1 \\
& =\underset{\boldsymbol{S}, \boldsymbol{S}^{\prime} \sim \mathcal{H}}{\mathbf{E}}\left[\exp \left(n \epsilon^{2} \boldsymbol{\delta}^{2}\right)\right]-1 .
\end{aligned}
$$

Since $\exp \left(n \epsilon^{2} \boldsymbol{\delta}^{2}\right)-1 \geq 0$

$$
\underset{\boldsymbol{S}, \boldsymbol{S}^{\prime} \sim \mathcal{H}}{\mathbf{E}}\left[\exp \left(n \epsilon^{2} \boldsymbol{\delta}\right)\right]-1=\int_{0}^{\infty} \underset{\boldsymbol{S}, \boldsymbol{S}^{\prime} \sim \mathcal{H}}{\mathbf{P}}\left[\exp \left(n \epsilon^{2} \boldsymbol{\delta}^{2}\right)-1 \geq t\right] d t .
$$

Since $\exp \left(n \epsilon^{2} \boldsymbol{\delta}^{2}\right)-1 \geq t$ is equivalent to

$$
\boldsymbol{r} \geq \frac{d}{4}+\frac{d}{4} \cdot\left(\frac{\log (1+t)}{n \epsilon^{2}}\right)^{\frac{1}{2}}
$$

it follows that

$$
d_{\chi^{2}}\left(\mathcal{D}_{n}, \text { Unif }_{d^{2}}^{\otimes n}\right) \leq \int_{0}^{\infty} \underset{\boldsymbol{S}, \boldsymbol{S}^{\prime} \sim \mathcal{H}}{\mathbf{P}}\left[\boldsymbol{r} \geq \frac{d}{4}+\frac{d}{4} \sqrt{f(t)}\right] d t,
$$

where $f(t)=\log (1+t) / n \epsilon^{2}$. 
Since $\boldsymbol{r}=\left|\boldsymbol{S} \cap \boldsymbol{S}^{\prime}\right|$ is invariant under permutations of $[d]$, it follows that $\boldsymbol{r}$ is distributed according to the hypergeometric distribution with $d / 2$ draws from a set of $d$ elements with $d / 2$ successes. If $\boldsymbol{X}$ is a random variable distributed according to the hypergeometric distribution with $m$ draws from a set of $N$ elements with $k$ successes, then (see e.g. [Ska13])

$$
\mathbf{P}\left[\frac{\boldsymbol{X}}{m} \geq \frac{k}{N}+s\right] \leq \exp \left(-2 s^{2} m\right) .
$$

Hence,

$$
\underset{\boldsymbol{S}, \boldsymbol{S}^{\prime} \sim \mathcal{H}}{\mathbf{P}}\left[\boldsymbol{r} \cdot \frac{2}{d} \geq \frac{1}{2}+t\right]=\underset{\boldsymbol{S}, \boldsymbol{S}^{\prime} \sim \mathcal{H}}{\mathbf{P}}\left[\boldsymbol{r} \geq \frac{d}{4}+\frac{d t}{2}\right] \leq \exp \left(-d t^{2}\right),
$$

whence,

$$
\underset{\boldsymbol{S}, \boldsymbol{S}^{\prime} \sim \mathcal{H}}{\mathbf{P}}\left[\boldsymbol{r} \geq \frac{d}{4}(\sqrt{f(t)}+1)\right] \leq \exp (-d f(t) / 4) .
$$

Therefore,

$$
\begin{aligned}
d_{\chi^{2}}\left(\mathcal{D}_{n}, \text { Unif }_{d^{2}}^{\otimes n}\right) & \leq \int_{0}^{\infty} \exp (-d f(t) / 4) d t \\
& =\int_{0}^{\infty} \exp \left(-\frac{d}{4 n \epsilon^{2}} \log (1+t)\right) d t \\
& =\int_{0}^{\infty}\left(\frac{1}{1+t}\right)^{c} d t, \\
& =\frac{1}{c-1},
\end{aligned}
$$

where $c=d / 4 n \epsilon^{2}$. Since $d_{\chi^{2}}\left(\mathcal{D}_{n}, \mathrm{Unif}_{d^{2}}^{\otimes n}\right) \geq 1 / 3$, it follows that $c \leq 4$, so $n \geq d / 16 \epsilon^{2}$. Therefore, $n=\Omega\left(d / \epsilon^{2}\right)$, as needed.

Let $d_{\mathrm{TV}}\left(-,{ }_{-}\right)$denote the total variation distance between probability distributions. Let $p \in \mathrm{CPD}_{d}$ and let $q$ be a distribution $\epsilon$-far from $\mathrm{CPD}_{d}$.

A testing algorithm $f:\left([d]^{2}\right)^{n} \rightarrow\{0,1\}$ for complete positivity determines a probability event $E \subseteq\left([d]^{2}\right)^{n}$ satisfying $p^{\otimes n}(E) \geq 2 / 3$ and $q^{\otimes n}(E) \leq 1 / 3$. Hence, $\operatorname{Unif}_{d^{2}}^{\otimes n}(E) \geq 2 / 3$ and, since $\mathcal{D}_{n}$ is supported on distributions $\epsilon$-far from $\mathrm{CPD}_{d}, \mathcal{D}_{n}(E) \leq 1 / 3$. Therefore, $d_{\mathrm{TV}}\left(\mathcal{D}_{n}, \mathrm{Unif}_{d^{2}}^{\otimes n}\right) \geq 1 / 3$ and the following corollary establishes the lower bound:

Corollary 3.2. If $d_{\mathrm{TV}}\left(\mathcal{D}_{n}, \mathrm{Unif}_{d^{2}}^{\otimes n}\right) \geq 1 / 3$, then $n=\Omega\left(d / \epsilon^{2}\right)$. 
Proof. For all distributions $\mu$ and $\nu, 2 d_{\mathrm{TV}}(\mu, \nu)^{2} \leq d_{\chi^{2}}(\mu, \nu)$. Hence,

$$
\left(d / 4 n \epsilon^{2}-1\right)^{-1} \geq d_{\chi^{2}}\left(\mathcal{D}_{n}, \mathrm{Unif}_{d^{2}}^{\otimes n}\right) \geq 2 d_{\mathrm{TV}}\left(\mathcal{D}_{n}, \mathrm{Unif}_{d^{2}}^{\otimes n}\right)^{2} \geq \frac{2}{9}
$$

where the first inequality is obtained in the proof of Proposition 3.1. Therefore, $n=\Omega\left(d / \epsilon^{2}\right)$.

\subsection{Hardness of learning completely positive distributions}

Given our $\Omega\left(d / \epsilon^{2}\right)$ lower bound for testing if a distribution $p$ on $[d] \times[d]$ is completely positive, it is natural to ask whether one can improve the trivial upper bound of $O\left(d^{2} / \epsilon^{2}\right)$. This trivial upper bound comes from fully learning $p$ to $\epsilon / 2$ accuracy in trace distance and then checking if the estimate is $\epsilon / 2$-close to CP. A natural strategy for improving the testing upper bound is the testing by learning method; if we could show an $o\left(d^{2} / \epsilon^{2}\right)$ upper bound for learning an unknown $p$ on $[d] \times[d]$ under the assumption that it is $\mathrm{CP}$, this would yield an improved testing upper bound. However, here we show that this is not possible.

Theorem 3.3. Let $\mathcal{L}$ be an algorithm that gets as input a parameter $0<\epsilon<\frac{1}{2}$ as well as access to $n$ samples from a completely positive probability distribution $p$ on $[d] \times[d]$. Suppose $\mathcal{L}$ is guaranteed to output a hypothesis distribution $\widehat{p}$ satisfying $d_{\mathrm{TV}}(p, \widehat{p}) \leq .1 \epsilon$ with probability at least $\frac{2}{3}$. Then $n \geq \Omega\left(d^{2} / \epsilon^{2}\right)$.

Proof. By the Fano inequality method, it suffices to construct completely positive distributions $p_{1}, \ldots, p_{N}$ on $[d] \times[d]$ with:

$$
\text { (i) } N \geq 2^{\Omega\left(d^{2}\right)} ; \quad \text { (ii) } d_{\mathrm{TV}}\left(p_{i}, p_{j}\right)>.1 \epsilon \text { for all } i \neq j ; \quad \text { (iii) } \mathrm{KL}\left(p_{i} \| p_{j}\right) \leq O\left(\epsilon^{2}\right) \text { for all } i \neq j \text {, }
$$

where KL(_ $\left.\|_{-}\right)$denotes Kullback-Leibler divergence.

To get started on this, let $\mathcal{I}$ denote the set of $\left(\begin{array}{l}d \\ 2\end{array}\right)$ "upper-triangular" pairs $\{(i, j) \in$ $[d] \times[d]: i<j\}$; similar to before we may assume, without of loss of generality, that $d$ is a multiple of 4 and hence $|\mathcal{I}|$ is even. Given $(i, j) \in \mathcal{I}$, let us define the probability distribution $q_{(i, j)}$ on $[d] \times[d]$ by:

$$
q_{(i, j)} \text { has probability mass } \frac{1}{4} \text { on each of }(i, i),(i, j),(j, i),(j, j) \text {. }
$$

Note that $q_{(i, j)}$ is a product distribution, namely the two-fold product of the distribution on $[d]$ that puts mass $\frac{1}{2}$ on $i$ and mass $\frac{1}{2}$ on $j$. Thus any mixture of $q_{(i, j)}$ 's is a completely positive distribution. 
Next, let $\mathcal{M}=\left(e_{1}, \ldots, e_{m}\right)$ denote an arbitrary perfect matching on $\mathcal{I}$, where $m=\frac{1}{2}\left(\begin{array}{l}d \\ 2\end{array}\right)$. If $e=\left(\left(i_{0}, j_{0}\right),\left(i_{1}, j_{1}\right)\right)$ is a typical matched pair from $\mathcal{M}$, define the following two completely positive distributions:

$$
r_{e}^{(0)}=\frac{1+\epsilon}{2} q_{\left(i_{0}, j_{0}\right)}+\frac{1-\epsilon}{2} q_{\left(i_{1}, j_{1}\right)}, \quad r_{e}^{(1)}=\frac{1-\epsilon}{2} q_{\left(i_{0}, j_{0}\right)}+\frac{1+\epsilon}{2} q_{\left(i_{1}, j_{1}\right)} .
$$

Next, given $\omega \in\{0,1\}^{m}$, define the following completely positive distribution:

$$
\widetilde{p}_{\omega}=\underset{k=1 \ldots m}{\operatorname{avg}} r_{e_{k}}^{\left(\omega_{k}\right)}
$$

That is, $\widetilde{p}_{\omega}$ is an equal mixture of some half of the the $r_{e}^{(\cdot)}$ distributions, with $\omega$ selecting which of $r_{e}^{(0 / 1)}$ is taken for each $e \in \mathcal{M}$.

Let us now consider the "diagonal" probability mass of the $\widetilde{p}_{\omega}$ distributions. First, since every $q_{(i, j)}$ puts $\frac{1}{2}$ of its probability mass on diagonal elements, the same is true of the $r_{e}^{(\cdot)}$ and $\widetilde{p}_{\omega}$ distributions. Furthermore, it is easy see that $\widetilde{p}_{\omega}(i, i)=\frac{1+\delta_{\omega}^{i}}{2 d}$ for numbers $\delta_{\omega}^{i} \in[-\epsilon, \epsilon]$ for all $i \in[d]$ and $\omega \in\{0,1\}^{m}$. Thus for each $\omega \in\{0,1\}^{m}$ we may construct a "correcting" distribution $u_{\omega}$ on $[d] \times[d]$, completely supported on diagonal elements with $u_{\omega}(i, i)=\frac{1-\delta_{\omega}^{i} / 2}{d}$, such that

$$
p_{\omega}:=\frac{1}{2} \widetilde{p}_{\omega}+\frac{1}{2} u_{\omega}
$$

has $p_{\omega}(i, i)=\frac{3}{4 d}$ for all $i \in[d]$. In other words, each $p_{\omega}$ has $\frac{3}{4}$ of its probability mass on the diagonal, and is uniform on the diagonal elements. Furthermore, the distributions $p_{\omega}$ are still completely positive, since every diagonal distribution is completely positive.

Our final collection of CP distributions $p_{1}, \ldots, p_{N}$ satisfying Equation (2) will be a subset of the $p_{\omega}$ 's. Since any two $p_{\omega}, p_{\omega^{\prime}}$ agree on the diagonal elements, we may calculate:

$$
d_{\mathrm{TV}}\left(p_{\omega}, p_{\omega^{\prime}}\right)=\sum_{(i, j) \in \mathcal{I}}\left|p_{\omega}(i, j)-p_{\omega^{\prime}}(i, j)\right|=\frac{1}{2} \underset{k=1 \ldots m}{\operatorname{avg}}\left\{\begin{array}{ll}
\frac{\epsilon}{2} & \text { if } \omega_{k} \neq \omega_{k}^{\prime} \\
0 & \text { if } \omega_{k}=\omega_{k}^{\prime}
\end{array}\right\}=\frac{\Delta\left(\omega, \omega^{\prime}\right)}{4 m} \epsilon
$$

where $\Delta(-,-)$ is Hamming distance. One may similarly compute

$$
\mathrm{KL}\left(p_{\omega} \| p_{\omega^{\prime}}\right)=\frac{\Delta\left(\omega, \omega^{\prime}\right)}{8 m} \epsilon \ln \left(\frac{1+\epsilon}{1-\epsilon}\right) \leq \frac{3 \Delta\left(\omega, \omega^{\prime}\right)}{8 m} \epsilon^{2}
$$

where the inequality used $\epsilon<\frac{1}{2}$. To complete the proof via Equation (2), it remains to choose a subset of $N=2^{\Omega(m)}=2^{\Omega\left(d^{2}\right)}$ elements of $\{0,1\}^{m}$ such that any two distinct chosen $\omega, \omega^{\prime}$ have $\Delta\left(\omega, \omega^{\prime}\right) \geq .4 m$. This is easily done via the probabilistic method, or an explicit error-correcting code. 


\section{Testing separability}

Let $d$ be a positive integer. As in the previous section, we may assume, without loss of generality, that $d$ is even.

Let $\mathcal{H}=\mathbb{C}^{d} \otimes \mathbb{C}^{d}$, let $\mathrm{U}(\mathcal{H})$ denote the set of unitary operators on $\mathcal{H}$, and recall that Sep denotes the set of separable states on $\mathcal{H}$. For all operators $T$ on $\mathcal{H}$, let $\|T\|_{p}$ denote the Schatten $p$-norm of $T$, viz. $\|T\|_{p}=\left(\operatorname{tr}\left(|T|^{p}\right)\right)^{\frac{1}{p}}$, where $|T|=\sqrt{T^{\dagger} T}$ is the absolute value of the operator $T$. Let $d_{\operatorname{tr}}(\varrho, \sigma)=\frac{1}{2}\|\varrho-\sigma\|_{1}$ denote the trace distance between quantum states $\varrho$ and $\sigma$.

We begin by defining a family of quantum states which are with high probability $O(\epsilon)$-far from Sep. For $0 \leq \epsilon \leq 1 / 2$, let $\mathrm{D}_{\epsilon}$ be the diagonal matrix on $\mathcal{H}$ defined by

$$
\mathrm{D}_{\epsilon}=\operatorname{diag}\left(\frac{1+2 \epsilon}{d^{2}}, \ldots, \frac{1+2 \epsilon}{d^{2}}, \frac{1-2 \epsilon}{d^{2}}, \ldots, \frac{1-2 \epsilon}{d^{2}}\right),
$$

where $\operatorname{tr}\left(D_{\epsilon}\right)=1$, and let $\mathcal{D}$ denote the family of all quantum states on $\mathcal{H}$ with the same spectrum as $\mathrm{D}_{\epsilon}$, viz. $\mathcal{D}=\left\{U \mathrm{D}_{\epsilon} U^{\dagger} \mid U \in \mathrm{U}(\mathcal{H})\right\}$.

Our lower bound will rely on the following theorem which follows immediately from [OW15, Lemma 2.22 and Theorem 4.2]:

Theorem 4.1. $\Omega\left(d^{2} / \epsilon^{2}\right)$ copies are necessary to test whether a quantum state $\varrho$ on $\mathcal{H}$ is the maximally mixed state or $\varrho \in \mathcal{D}$.

If $\boldsymbol{U}$ is a random unitary on $\mathcal{H}$ distributed according to the Haar measure, then $\varrho=$ $\boldsymbol{U} \mathrm{D}_{\epsilon} \boldsymbol{U}^{\dagger}$ is a random element of $\mathcal{D}$. This induced probability measure is invariant under conjugation by a fixed unitary: for all $V \in \mathrm{U}(\mathcal{H}), V \varrho V^{\dagger}$ has the same distribution as $\varrho$. We want to show the following:

Lemma 4.2. There is a universal constant $C_{0}$ such that for all $C_{0} / \sqrt{d} \leq \epsilon \leq 1 / 2$, the following holds when $\varrho=\boldsymbol{U} \mathrm{D}_{\epsilon} \boldsymbol{U}^{\dagger}$ is a uniformly random state in $\mathcal{D}$ :

$$
\mathbf{P}\left[\forall \sigma \in \operatorname{Sep},\|\varrho-\sigma\|_{1} \geq 2 \epsilon\right] \geq \frac{2}{3}
$$

As $\epsilon$ tends to zero, the elements of $\mathcal{D}$ get closer to the maximally mixed state and eventually become separable, by the Gurvits-Barnum theorem [GB02]. Indeed, if $\epsilon \leq 1 /\left(2 \sqrt{d^{2}-1}\right)$, then $\mathcal{D} \subseteq$ Sep. Hence, some assumption on $\epsilon$ is necessary for Lemma 4.2 to hold.

Lemma 4.2 and Theorem 4.1 easily imply the desired lower bound:

Theorem 4.3. Let $\varrho$ be a quantum state on $\mathbb{C}^{d} \otimes \mathbb{C}^{d}$ and let $\epsilon=\Omega(1 / \sqrt{d})$. Testing if $\varrho$ is separable or $\epsilon$-far from Sep in trace distance requires $\Omega\left(d^{2} / \epsilon^{2}\right)$ copies of $\varrho$. 
Proof. Let $\left\{E_{0}, E_{1}\right\}$ be a measurement corresponding to a separability testing algorithm using $n$ copies of $\varrho$. To apply the lower bound in Theorem 4.1 , we use $\left\{E_{0}, E_{1}\right\}$ to define an algorithm that decides w.h.p. if a state $\varrho$ is equal to the maximally mixed state $\frac{\mathbb{1}}{d^{2}}$ or $\varrho \in \mathcal{D}$.

Let $\varrho^{\otimes n}$ be given with either $\varrho \in \mathcal{D}$ or $\varrho=\frac{\mathbb{1}}{d^{2}}$. Note that, for all $\varrho \in \mathcal{D}, d_{\text {tr }}\left(\varrho, \frac{\mathbb{1}}{d^{2}}\right) \geq \epsilon$ holds. Let $\boldsymbol{U}$ be a random unitary. If $\varrho$ is the maximally mixed state, then $V \varrho V^{\dagger}=\varrho$ for all $V \in \mathrm{U}(\mathcal{H})$, so $\left(\boldsymbol{U} \varrho \boldsymbol{U}^{\dagger}\right)^{\otimes n}=\varrho^{\otimes n}$. Otherwise, $\boldsymbol{U} \varrho \boldsymbol{U}^{\dagger}$ is a random state in $\mathcal{D}$.

Applying the separability test $\left\{E_{0}, E_{1}\right\}$ to $\boldsymbol{U} \varrho \boldsymbol{U}^{\dagger}$, we have that:

(i) if $\boldsymbol{U} \varrho \boldsymbol{U}^{\dagger}=\varrho=\frac{\mathbb{1}}{d^{2}}$, then $\boldsymbol{U} \varrho \boldsymbol{U}^{\dagger}$ is separable, so

$$
\operatorname{tr}\left(\left(\boldsymbol{U} \boldsymbol{U}^{\dagger}\right)^{\otimes n} E_{1}\right)=\operatorname{tr}\left(\varrho^{\otimes n} E_{1}\right) \geq \frac{2}{3} .
$$

(ii) if $\varrho \in \mathcal{D}$, then the probability of error is

$$
\begin{aligned}
\underset{\boldsymbol{U}}{\mathbf{E}} \operatorname{tr}\left(\left(\boldsymbol{U} \varrho \boldsymbol{U}^{\dagger}\right)^{\otimes n} E_{1}\right) & \leq \mathbf{P}\left[\boldsymbol{U} \varrho \boldsymbol{U}^{\dagger} \text { is } \epsilon \text {-close to Sep }\right]+\mathbf{P}\left[\text { test fails } \mid \boldsymbol{U} \varrho \boldsymbol{U}^{\dagger} \text { is } \epsilon \text {-far from Sep }\right] \\
& \leq \frac{1}{3}+\frac{1}{3} \cdot \frac{2}{3}=\frac{5}{9},
\end{aligned}
$$

where the second inequality follows from Lemma 4.2.

Thus, using the separability test, we can distinguish w.h.p. between $\varrho=\frac{\mathbb{1}}{d^{2}}$ and $\varrho \in \mathcal{D}$ using $n$ copies of $\varrho$. Therefore, by Theorem $4.1, n=\Omega\left(d^{2} / \epsilon^{2}\right)$.

It remains to show that Lemma 4.2 holds. Its proof relies on two main facts: first, that Sep is approximated by a polytope with $\exp (O(d)))$ vertices which are separable pure states; and, second, that a random element of $\mathcal{D}$ is $\epsilon$-far from a fixed pure state except with probability $\exp (-O(d))$.

The first fact follows from the next lemma which is a rephrasing of [AS17b, Lemma 9.4]:

Lemma 4.4. There exists a constant $C>0$ such that, for every dimension $d$, there is a family $\mathcal{N}$ of pure product states on $\mathcal{H}$ (i.e. states of the form $|x \otimes y\rangle\langle x \otimes y|$ with $x, y \in \mathbb{C}^{d}$ ) with $|\mathcal{N}| \leq C^{d}$ satisfying

$$
\operatorname{conv}(\mathcal{N} \cup-\mathcal{N}) \subseteq \operatorname{Sep}_{ \pm} \subseteq 2 \operatorname{conv}(\mathcal{N} \cup-\mathcal{N})
$$

Now, we wish to upper bound the probability that a random element of $\mathcal{D}$ is $\epsilon$-far from a fixed pure state. The following result provides a sufficient condition for a state $\sigma$ on $\mathcal{H}$ to be $\epsilon$-far from a state $\varrho \in \mathcal{D}$ :

Proposition 4.5. Let $\varrho \in \mathcal{D}$ be arbitrary and let $W=\frac{\mathbb{1}}{d^{2}}-\varrho$. For all quantum states $\sigma$ on $\mathcal{H}$, if $\operatorname{tr}(\sigma W) \geq-\epsilon\|W\|_{\infty}$, then $\|\varrho-\sigma\|_{1} \geq \epsilon$. 
Proof. Note that

$$
\begin{aligned}
\operatorname{tr}(\varrho W) & =\frac{1}{d^{2}}-\operatorname{tr}\left(\varrho^{2}\right)=\frac{1}{d^{2}}-\frac{1+4 \epsilon^{2}}{d^{2}}=-\frac{4 \epsilon^{2}}{d^{2}}, \\
\|W\|_{\infty} & =\left\|\frac{\mathbb{1}}{d^{2}}-\mathrm{D}_{\epsilon}\right\|_{\infty}=\frac{2 \epsilon}{d^{2}} .
\end{aligned}
$$

By Hölder's inequality for matrices, $\operatorname{tr}((\sigma-\varrho) W) \leq\|\sigma-\varrho\|_{1} \cdot\|W\|_{\infty}$. Hence,

$$
\|\sigma-\varrho\|_{1} \geq \frac{\operatorname{tr}(\sigma W)-\operatorname{tr}(\varrho W)}{\|W\|_{\infty}}=2 \epsilon+\frac{\operatorname{tr}(\sigma W)}{\|W\|_{\infty}} .
$$

When $\sigma=|x\rangle\langle x|$ with $x \in \mathcal{H}$ and $\varrho=U \mathrm{D}_{\epsilon} U^{\dagger}$, we have

$$
\begin{aligned}
\operatorname{tr}(|x\rangle\langle x| W) & =\langle x|W| x\rangle \\
& =\left\langle x\left|\left(\frac{\mathbb{1}}{d^{2}}-U \mathrm{D}_{\epsilon} U^{\dagger}\right)\right| x\right\rangle \\
& =\left\langle x\left|U\left(\frac{\mathbb{1}}{d^{2}}-\mathrm{D}_{\epsilon}\right) U^{\dagger}\right| x\right\rangle \\
& =\|W\|_{\infty} \cdot\left\langle x\left|U Z U^{\dagger}\right| x\right\rangle,
\end{aligned}
$$

where $\mathbf{Z}=\operatorname{diag}(-1, \ldots,-1,1, \ldots, 1)$ is just $\mathbb{1} / d^{2}-\mathrm{D}_{\epsilon}$ divided by $\|W\|_{\infty}$. Hence, $\| \varrho-$ $|x\rangle\langle x| \|_{1} \geq \epsilon$ holds if $\left\langle x\left|U Z U^{\dagger}\right| x\right\rangle \geq-\epsilon$.

Since we are interested in the case when $\varrho=\boldsymbol{U} \mathrm{D}_{\epsilon} \boldsymbol{U}^{\dagger}$ is random, it suffices to show that $\left\langle x\left|\boldsymbol{U} Z \boldsymbol{U}^{\dagger}\right| x\right\rangle$ concentrates in the interval $[-\epsilon, \epsilon]$. This fact follows easily from the next lemma:

Lemma 4.6. Let $k$ be a positive even integer. If $\boldsymbol{u} \in \mathbb{C}^{k}$ is a uniformly random unit vector, then, for sufficiently large $k$,

$$
\mathbf{P}\left[|\langle\boldsymbol{u}|Z| \boldsymbol{u}\rangle| \geq \frac{1}{2} c k^{-1 / 4}\right] \leq 4 \exp \left(-\sqrt{k} c^{2} / 8\right),
$$

where $Z=\operatorname{diag}(1, \ldots, 1,-1, \ldots,-1)$ is a $k \times k$ diagonal matrix with $\operatorname{tr}(Z)=0$ and $c$ may be any positive constant.

Proof. Let $\boldsymbol{u}=\left(\boldsymbol{a}_{1}+i \boldsymbol{b}_{1}, \ldots, \boldsymbol{a}_{k}+i \boldsymbol{b}_{k}\right) \in \mathbb{C}^{k}$ be a uniformly random unit vector with $\boldsymbol{a}_{1}, \ldots, \boldsymbol{a}_{k}, \boldsymbol{b}_{1}, \ldots, \boldsymbol{b}_{k} \in \mathbb{R}$ and let $\boldsymbol{v} \in \mathbb{R}^{2 k}$ be defined by

$$
\boldsymbol{v}=\left(\boldsymbol{a}_{1}, \ldots, \boldsymbol{a}_{\frac{k}{2}}, \boldsymbol{b}_{1}, \ldots, \boldsymbol{b}_{\frac{k}{2}}, \boldsymbol{a}_{\frac{k}{2}+1}, \ldots, \boldsymbol{a}_{k}, \boldsymbol{b}_{\frac{k}{2}+1}, \ldots, \boldsymbol{b}_{k}\right) .
$$


Let $D$ be the $2 k \times 2 k$ diagonal matrix $D=\operatorname{diag}(1, \ldots, 1,-1, \ldots,-1)$ with $\operatorname{tr}(D)=0$. Thus, $\boldsymbol{v}$ is a uniformly random real unit vector such that $\langle\boldsymbol{v}|D| \boldsymbol{v}\rangle=\langle\boldsymbol{u}|Z| \boldsymbol{u}\rangle$.

Let $\boldsymbol{x}_{1}, \ldots, \boldsymbol{x}_{k}, \boldsymbol{y}_{1}, \ldots, \boldsymbol{y}_{k} \in \mathbb{R}$ be $2 k$ standard Gaussian random variables. Let $\boldsymbol{X}=$ $\boldsymbol{x}_{1}^{2}+\cdots+\boldsymbol{x}_{k}^{2}$ and $\boldsymbol{Y}=\boldsymbol{y}_{1}^{2}+\cdots+\boldsymbol{y}_{k}^{2}$. By the rotational symmetry of multivariate Gaussian random variables, $\boldsymbol{v}$ has the same distribution as

$$
\frac{\left(\boldsymbol{x}_{1}, \ldots, \boldsymbol{x}_{k}, \boldsymbol{y}_{1}, \ldots, \boldsymbol{y}_{k}\right)}{\sqrt{\boldsymbol{X}+\boldsymbol{Y}}}
$$

Hence, $\langle\boldsymbol{v}|D| \boldsymbol{v}\rangle$ and $\frac{\boldsymbol{X}-\boldsymbol{Y}}{\boldsymbol{X}+\boldsymbol{Y}}$ have the same distribution. Since $\boldsymbol{X}$ and $\boldsymbol{Y}$ are independent $\chi^{2}$ random variables with $k$ degrees of freedom each, it holds that (see e.g. [Wai19, Example 2.11])

$$
\mathbf{P}\left[\left|\frac{\boldsymbol{X}}{k}-1\right| \geq t\right] \leq 2 \exp \left(-k t^{2} / 8\right)
$$

for all $t \in(0,1)$ and similarly for $Y$. Hence, for $t=c k^{-1 / 4}$, we have $\mathbf{P}\left[|\boldsymbol{X}-k| \geq c k^{3 / 4}\right] \leq$ $2 \exp \left(-\sqrt{k} c^{2} / 8\right)$.

If $|\boldsymbol{X}-k|<c k^{3 / 4}$ and $|\boldsymbol{Y}-k|<c k^{3 / 4}$, then, for $k$ sufficiently large,

$$
|\langle\boldsymbol{v}|D| \boldsymbol{v}\rangle|=\frac{|\boldsymbol{X}-\boldsymbol{Y}|}{\boldsymbol{X}+\boldsymbol{Y}} \leq \frac{2 c k^{3 / 4}}{2 k-2 c k^{3 / 4}}=\frac{c}{k^{1 / 4}-1}<\frac{1}{2} c k^{-1 / 4}
$$

Hence, $\mathbf{P}\left[|\langle\boldsymbol{v}|D| \boldsymbol{v}\rangle|<\frac{1}{2} c k^{-1 / 4}\right] \geq 1-4 \exp \left(-\sqrt{k} c^{2} / 8\right)$.

If $\boldsymbol{U}$ is a random unitary distributed according to the Haar measure on $\mathrm{U}(\mathcal{H})$ and $x \in \mathcal{H}$ is a fixed unit vector, then $\boldsymbol{u}=\boldsymbol{U}|x\rangle$ is a uniformly random unit vector in $\mathcal{H}$. Hence, we can apply Lemma 4.6 to $|\langle\boldsymbol{u}|\mathbf{Z}| \boldsymbol{u}\rangle|$ to get

$$
\mathbf{P}\left[\left|\left\langle x\left|\boldsymbol{U} Z \boldsymbol{U}^{\dagger}\right| x\right\rangle\right| \geq \epsilon\right] \leq 4 \exp \left(-d c^{2} / 8\right),
$$

where $c$ is an arbitrary positive constant and $\epsilon \geq \frac{1}{2} c d^{-1 / 2}$.

We now have all the elements needed to prove Lemma 4.2:

Proof of Lemma 4.2. Let $\varrho=U \mathrm{D}_{\epsilon} \boldsymbol{U}^{\dagger}$ be a uniformly random element of $\mathcal{D}$ and let $\boldsymbol{W}=$ $\frac{\mathbb{1}}{d^{2}}-\varrho$. Thus, assuming $\epsilon \geq c d^{-1 / 2}$,

$$
\begin{aligned}
\mathbf{P}[\forall \sigma & \left.\in \operatorname{Sep}, d_{\mathrm{TV}}(\varrho, \sigma) \geq \epsilon\right] \\
& =\mathbf{P}\left[\forall \sigma \in \operatorname{Sep}, \quad\|\boldsymbol{\varrho}-\sigma\|_{1} \geq 2 \epsilon\right] \\
& \geq \mathbf{P}\left[\forall \sigma \in \operatorname{Sep}, \quad \operatorname{tr}(\sigma \boldsymbol{W}) \geq-2 \epsilon\|\boldsymbol{W}\|_{\infty}\right]
\end{aligned}
$$




$$
\begin{aligned}
& \geq \mathbf{P}\left[\forall \sigma \in 2 \operatorname{conv}(\mathcal{N} \cup-\mathcal{N}), \operatorname{tr}(\sigma \boldsymbol{W}) \geq-2 \epsilon\|\boldsymbol{W}\|_{\infty}\right] \quad \text { (by Lemma 4.4) } \\
& =\mathbf{P}\left[\forall|x\rangle\langle x| \in \mathcal{N} \cup-\mathcal{N}, 2 \operatorname{tr}(|x\rangle\langle x| \boldsymbol{W}) \geq-2 \epsilon\|\boldsymbol{W}\|_{\infty}\right] \quad \text { (by convexity) } \\
& =\mathbf{P}\left[\forall|x\rangle\left\langle x|\in \mathcal{N},|\left\langle x\left|\boldsymbol{U} Z \boldsymbol{U}^{\dagger}\right| x\right\rangle\right| \leq \epsilon\right] \quad \text { (by Equation (3)) } \\
& \geq 1-\sum_{|x\rangle\langle x| \in \mathcal{N}} \mathbf{P}\left[\left|\left\langle x\left|\boldsymbol{U} Z \boldsymbol{U}^{\dagger}\right| x\right\rangle\right|>\epsilon\right] \quad \text { (by the union bound) } \\
& \geq 1-|\mathcal{N}| \cdot 4 \exp \left(-d c^{2} / 8\right) \quad \text { (by Equation (4)) } \\
& =1-4 \exp \left(d\left(\log C-c^{2} / 8\right)\right) \quad\left(\text { since }|\mathcal{N}|=C^{d}\right) .
\end{aligned}
$$

Hence, if $c=\sqrt{8(\log C+1)}$, then

$$
\begin{aligned}
\mathbf{P}\left[\forall \sigma \in \operatorname{Sep}, d_{\mathrm{TV}}(\boldsymbol{\varrho}, \sigma) \geq \epsilon\right] & \geq 1-4 \exp \left(d\left(\log C-c^{2} / 8\right)\right) \\
& =1-4 \exp (-d) \\
& \geq \frac{2}{3},
\end{aligned}
$$

for $d \geq \log 12$.

\section{References}

[AS17a] Guillaume Aubrun and Stanisł aw Szarek. Dvoretzky's theorem and the complexity of entanglement detection. Discrete Analysis, pages Paper No. 1, 20, 2017. 2

[AS17b] Guillaume Aubrun and Stanisław Szarek. Alice and Bob Meet Banach. American Mathematical Society, 2017. 7, 15

[Dia77] Persi Diaconis. Finite forms of de Finetti's theorem on exchangeability. Synthese, 36(2):271-281, October 1977. 2

[DPS04] Andrew C. Doherty, Pablo A. Parrilo, and Federico M. Spedalieri. Complete family of separability criteria. Physical Review A, 69(2), Feb 2004. 2

[GB02] Leonid Gurvits and Howard Barnum. Largest separable balls around the maximally mixed bipartite quantum state. Physical Review A, 66(6), Dec 2002. 14

[GM12] Bernd Gärtner and Jiří Matoušek. Approximation Algorithms and Semidefinite Programming. Springer Berlin Heidelberg, 2012. 4 
[GT09] Otfried Gühne and Géza Tóth. Entanglement detection. Physics Reports, 474(16):1-75, Apr 2009. 3

[Gur04] Leonid Gurvits. Classical complexity and quantum entanglement. Journal of Computer and System Sciences, 69(3):448-484, Nov 2004. 2

[HHHH09] Ryszard Horodecki, Paweł Horodecki, Michał Horodecki, and Karol Horodecki. Quantum entanglement. Reviews of Modern Physics, 81(2):865-942, Jun 2009. 3

$\left[\mathrm{HHJ}^{+}\right.$16] Jeongwan Haah, Aram W. Harrow, Zhengfeng Ji, Xiaodi Wu, and Nengkun Yu. Sample-optimal tomography of quantum states. In Proceedings of the 48th Annual ACM SIGACT Symposium on Theory of Computing - STOC 2016. ACM Press, 2016. 2

[MdW16] Ashley Montanaro and Ronald de Wolf. A survey of quantum property testing. Theory of Computing, 1(1):1-81, 2016. 3

[MM62] John E. Maxfield and Henryk Minc. On the matrix equation $X^{\prime} X=A$. Proceedings of the Edinburgh Mathematical Society, 13(02):125, 1962. 5

[OW15] Ryan O'Donnell and John Wright. Quantum spectrum testing. In Proceedings of the 4 7th Annual ACM SIGACT Symposium on Theory of Computing - STOC 2015. ACM Press, 2015. 3, 14

[OW16] Ryan O'Donnell and John Wright. Efficient quantum tomography. In Proceedings of the 48th Annual ACM SIGACT Symposium on Theory of Computing - STOC 2016. ACM Press, 2016. 2

[Pan08] Liam Paninski. A coincidence-based test for uniformity given very sparsely sampled discrete data. IEEE Transactions on Information Theory, 54(10):4750-4755, Oct 2008. 3

[Ska13] Matthew Skala. Hypergeometric tail inequalities: ending the insanity. 2013. Technical report, arXiv:1311.5939. 11

[Wai19] Martin J. Wainwright. High-Dimensional Statistics. Cambridge University Press, 2019. 17

[Wat18] John Watrous. The Theory of Quantum Information. Cambridge University Press, 2018. 6 